\title{
Obesity and Density of the Crystalline Lens: Revisiting a Growing Dilemma
}

\author{
Seyed-Farzad Mohammadi ${ }^{a}$ Mohsen Afarideh ${ }^{a}$ Hadi Z. Mehrjardia, b \\ Sara Mirhadi ${ }^{a}$ \\ ${ }^{a}$ Eye Research Center, Farabi Eye Hospital, Tehran University of Medical Sciences, and \\ bStudents' Scientific Research Center, Tehran University of Medical Sciences, Tehran, Iran
}

\begin{abstract}
What Is It about?
Obesity has been suggested to increase the risk of age-related cataractogenesis (ARC). The rationale behind this increased risk has been attributed to the metabolic effects of obesity on the crystalline lens density. Previous estimations of ARC focused around subjective clinical measurement of lens opacity by the Lens Opacities Classification System III (LOCS III). The recent availability of the Pentacam lens optical densitometry based on the Scheimpflug principle has provided objective quantification of lens optical density (LOD) in an easy and reproducible manner. The present study investigates potential correlations of body mass index as the indicator of obesity with higher LOCS III and LOD readings as parameters of ARC in a cohort of middle-aged healthy individuals.
\end{abstract}

\section{Keywords}

Obesity · Body mass index · Crystalline lens density · Age-related cataract · Pentacam .

Scheimpflug imaging

\begin{abstract}
Background/Aims: Obesity is believed to accelerate age-related cataractogenesis through various biomechanisms. On the contrary, there are also studies advocating the protective role of obesity against the cataract formation process. We investigate the correlation of body mass index (BMI) as a measure for obesity with crystalline optical lens density and opacity in a healthy adult population. Methods: In a cross-sectional setting, 93 consecutive disease-free adult individuals who were working staff of a university-based hospital were assessed for the association between crystalline lens density and opalescence [measured by the objective Pentacam HR lens densitometry and subjective Lens Opacity Classification System III (LOCS III), respectively] with the degree of obesity as defined by BMI. Results: LOCS III and crystalline lens density readings were positively correlated [Spearman rho CC ( $p$ value $)=0.224(0.034)$ ] However, we found neither LOCS III nor crystalline lens density to be correlated with BMI 
[Spearman rho CC $=-0.008(p=0.943)$ and $-0.062(p=0.560)$, respectively]. Conclusions: Results from the present study indicate a lack of association between obesity and densitometry of the crystalline in the adult population group. Further studies are required to confirm the order of causality and pathogenesis of this finding.

(C) 2017 The Author(s)

Published by S. Karger AG, Basel

\section{Introduction}

Obesity may accelerate age-related cataractogenesis (ARC), although the exact mechanisms are poorly understood [1-3]. Increased oxidative stress caused by higher circulating leptin levels of obese subjects is involved in the development and/or progression of cataract. Obesity is a low-grade systemic inflammation with elevated levels of C-reactive protein and proinflammatory cytokines that are associated with increased risk of cataract formation/ progression [3]. In addition, obesity directly or indirectly influences the osmotic stress and nonenzymatic glycation of lens proteins [4]. A recent study found that intralenticular sorbitol accumulation beyond a threshold level leads to the increased risk of cataract formation in WNIN-obese rats [5]. Finally, obesity is potentially linked to ARC secondary to its comorbid cardiometabolic conditions, including diabetes [6], dyslipidemia [7], and hypertension [8], which themselves are known risk factors of ARC $[9,10]$. The metabolic syndrome, a condition characterized by the following 5 components: central obesity, hyperglycemia, hypertriglyceridemia, hypertension, and reduced serum high-density lipoprotein cholesterol, has been associated with different types of ARC [11-13]. Therefore, it has been suggested that the association of ARC is stronger with central obesity than with general obesity [14].

ARC is routinely diagnosed in clinic settings by red reflex evaluation or slit-lamp examinations using the Lens Opacity Classification System III (LOCS III) grading [15]. This is because aging of the crystalline lens is almost synonymous with ARC, and nuclear sclerosis is a well-established component of lens senescence. Previous studies focused on the association between ARC and obesity using the subjective LOCS III measures. Recent advances in techniques have provided objective and precise optical densitometry of the crystalline lens. The Pentacam HR technology (Oculus, Wetzlar, Germany) gives an objective and reproducible lens optical density (LOD) profile using the Scheimpflug principle [16-18], providing an evenly focused representation of a section of the lens because the plane of film parallels the optical section through the lens $[19,20]$. LOD has been shown to be associated with diabetes [21], nutritional elements [22], smoking, and sun exposure [23], as established risk factors of ARC. In this paper, we aimed to unveil possible associations among LOCS III, LOD, and body mass index (BMI) as a measure of obesity in a cohort of disease-free middle-aged individuals.

\section{Patients and Results}

In our hospital-based cross-sectional survey, the correlation of LOCS III subjective clinical grading and LOD (objectively quantified by Pentacam HR; Oculus, Wetzlar, Germany; Fig. 1a) with BMI was assessed in the right eyes of 93 disease-free subjects [59\% females, average age: $45.1 \pm 4.6$ years (range: $40-55$ )] who were staff of a tertiary-level academic center (Farabi Eye Hospital, Tehran University of Medical Sciences) and fully met the eligibility criteria of the study (see Table 1 for details on the eligibility criteria). The Research Council of Tehran University of Medical Sciences had previously reviewed and approved the study protocol. In 


\section{Blionetliche 細们}

Fig. 1. a Crystalline lens densitometry was performed in 3D by placing the cylinder body with a volume of $1.6 \mathrm{~mm}^{3}$ in the pupil center and in the anterior outer cortex of the subcapsular region (z-axis position at $-0.1 \mathrm{~mm}$ ). b Scatter diagram of BMI versus crystalline LOD suggesting no significant association. (c) 2017 The Author(s). Published by S. Karger AG, Basel www.karger.com/bmh

Mohammadi et al.: Obesity and Density of the Crystalline Lens: Revisiting a Growing Dilemma

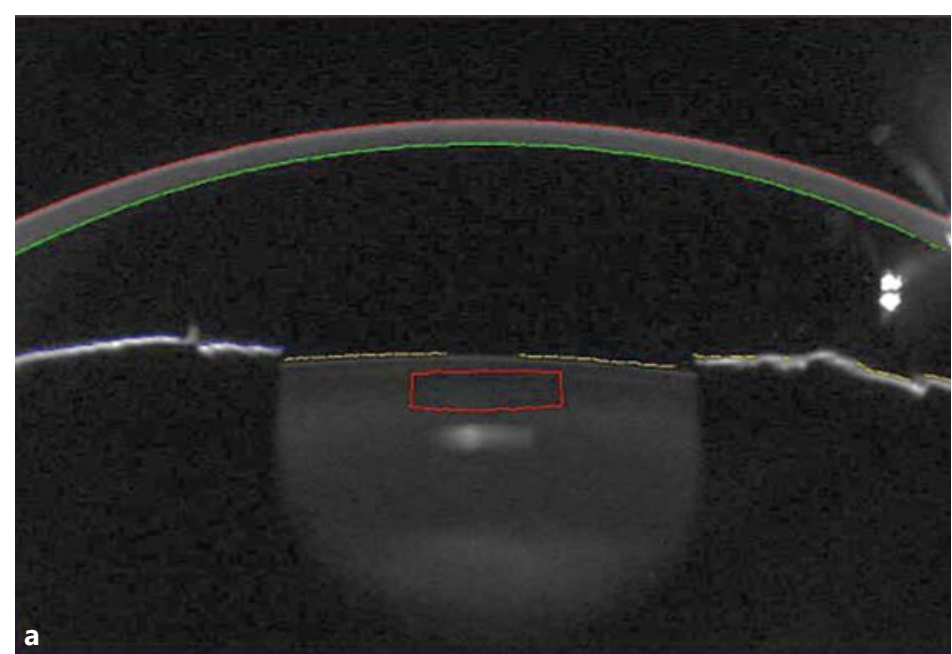

a

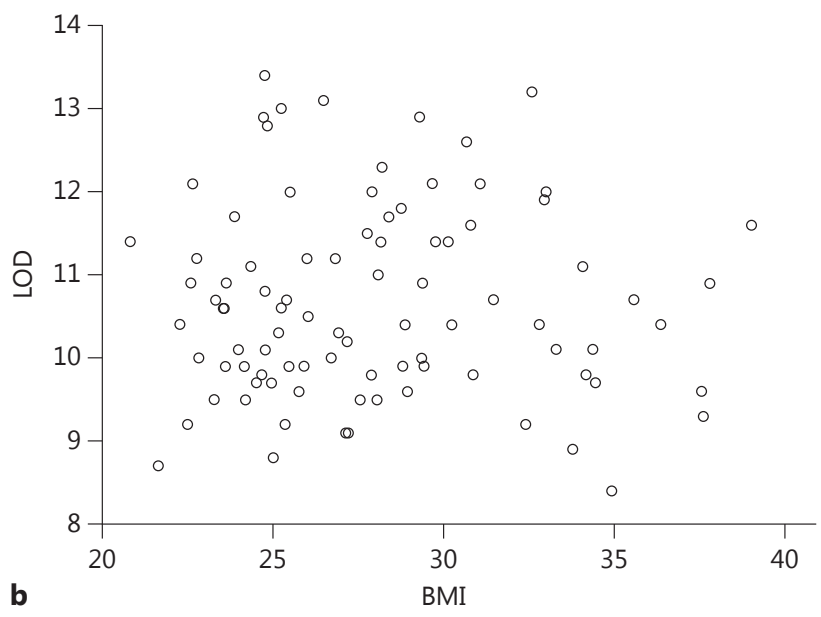

Table 1. Eligibility criteria of the study participants

Inclusion criteria

40-70-year-old nonphysician staff of Farabi Eye Hospital

Apparently healthy individuals without prior history of cataract

Exclusion criteria

Recent onset cataract

Diabetes mellitus

Hyperlipidemia

Use of simvastatin, levothyroxine, topical drugs and/or multivitamin

High myopia

LOCS III grade $>3$

Angle closure glaucoma (primary or secondary)

Subjects being residents of significantly higher altitude areas outside the Tehran metropolitan area compared with mean city altitude

Subjects wearing eyeglasses/sunglasses (UV protected) outdoor

Subjects wearing contact lenses outdoor

Primarily, 115 healthy subjects were selected to participate, of which 22 were excluded from the final analysis (total number of study participants $=93$ ). 
(C) 2017 The Author(s). Published by S. Karger AG, Basel www.karger.com/bmh

Mohammadi et al.: Obesity and Density of the Crystalline Lens: Revisiting a Growing Dilemma

Table 2. Associations of Scheimpflug LOD

\begin{tabular}{|c|c|c|c|}
\hline Factors & $\begin{array}{l}\text { Baseline, } \\
\text { mean (range)/n (\%) }\end{array}$ & $\begin{array}{l}\text { Scheimpflug } \mathrm{LOD}^{4} \\
\text { mean } \pm \mathrm{SD} / \text { coefficient }\end{array}$ & $p$ value $^{5}$ \\
\hline Age, years & $45.1(40-55)$ & $-/ 0.156$ & $0.139 *$ \\
\hline BMI & $27.8(20.8-39.0)$ & $-/-0.062$ & $0.560^{*}$ \\
\hline \multicolumn{4}{|l|}{ Gender } \\
\hline male & $38(41)$ & $10.8 \pm 1.2$ & \multirow[t]{2}{*}{0.302} \\
\hline female & 55 (59) & $10.6 \pm 1.1$ & \\
\hline \multicolumn{4}{|l|}{ Smoking } \\
\hline nonsmoker/occasional smoker & $78(84)$ & $10.7 \pm 1.2$ & \multirow[t]{2}{*}{0.407} \\
\hline current smoker & $15(16)$ & $10.5 \pm 0.7$ & \\
\hline \multicolumn{4}{|l|}{ Outdoor time estimate $^{1}$} \\
\hline low & $72(77)$ & $10.5 \pm 1.1$ & \multirow[t]{2}{*}{0.028} \\
\hline high & $21(23)$ & $11.2 \pm 1.3$ & \\
\hline \multicolumn{4}{|l|}{ Family history of cataract surgery ${ }^{2}$} \\
\hline negative & $44(47)$ & $10.6 \pm 1.1$ & \multirow[t]{2}{*}{0.424} \\
\hline positive & $49(53)$ & $10.8 \pm 1.2$ & \\
\hline \multicolumn{4}{|l|}{ Iris color } \\
\hline brown or black & $82(88)$ & $10.7 \pm 1.2$ & \multirow[t]{2}{*}{0.098} \\
\hline hazel, green, or blue & $11(12)$ & $11.5 \pm 0.9$ & \\
\hline \multicolumn{4}{|l|}{ LOCS III grading ${ }^{3}$} \\
\hline 1 & $47(50)$ & $10.49 \pm 1.2$ & \multirow{3}{*}{$0.034^{* *}$} \\
\hline 2 & $19(20)$ & $10.62 \pm 1.1$ & \\
\hline 3 & $27(30)$ & $11.11 \pm 1.2$ & \\
\hline
\end{tabular}

Prior to enrollment, subjects were asked on possible alcohol consumption, smoking, physical activity, iris color, complexion, sun exposure, and family history of cataract before the age of 50 years in their first-degree relatives.

${ }^{1}$ The outdoor time estimate was determined by occupation-attributed sun exposure and physical exercise.

${ }^{2}$ Family history of cataract surgery: history of cataract extraction in first-degree relatives.

${ }^{3}$ LOCS III: lens opacities classification system III [as among our population some categories of LOCS III were sparse, we combined them into 3 categories. By doing this, we tried to decrease the specificity (validity) and increase the sensitivity (reliability)].

${ }^{4}$ Scheimpflug lens optical density data did not significantly deviate from the normal distribution (Kolmogorov-Smirnov test; $p=0.349$ ).

${ }^{5}$ Student $t$ test or ANOVA was used for categorical factors.

* Due to lack of a linear association, the Spearman rho test was used to measure the magnitude and significance of correlations. ${ }^{* *}$ The Spearman rho correlation coefficient for LOCS III and LOD was 0.224.

accordance with the tenets of the Declaration of Helsinki, the informed consents of all participants were obtained for ethical considerations.

Complete ocular examinations were carried out by the same anterior segment specialist (S.-F.M.). Clinical LOCS III grading was employed to determine lens opacity. Participants were then treated with Tropicamid eye drop (dilated pupil), and LOD was measured by Pentacam HR technology (Oculus, Wetzlar, Germany), based on the Scheimpflug imaging technique. The Pentacam rotating Scheimpflug imaging system captures up to 50 slices in a single $180^{\circ}$ sweep around the central axis of the lens in approximately $2 \mathrm{~s}$, thereby providing a global $360^{\circ}$ evaluation of the crystalline lens density and avoiding the need to acquire multiple scans at different meridians as opposed to single-meridian shots in previous applications of the Scheimpflug technique by slit cameras [24]. The density (light transmittance) of the crystalline lens becomes visible by the blue light illumination with superb quality Scheimpflug images. A user-defined body is placed onto the crystalline lens and the reference point for 
centering the body is the pupil's center. Average and maximum density readings were measured by Pentacam using the user-selected evaluation body parallel to the axis.

Statistical analyses were performed using SPSS 19 software (IBM Corporation, New York, NY, USA) and findings are expressed as mean \pm standard deviation or ranges. Normality of data was previously tested using the Kolmogorov-Smirnov test. Correlates of continuous outcomes with categorical variables with normal distribution were performed by Student $t$ tests and analysis of variance (ANOVA) with a Bonferroni correction. Bivariate correlations were performed by Spearman rank correlation coefficient. In all tests, a $p$ value $<0.05$ was considered statistically significant.

Table 2 demonstrates the baseline characteristics of the studied population. Of note, outdoor time estimate was the only assessed risk factor to significantly correlate with LOD readings ( $11.2 \pm 1.3$ vs. $10.5 \pm 1.1$ for high vs. low outdoor exposures, $p=0.028)$. Although the LOCS III and LOD readings were positively correlated [Spearman's rho CC $=0.224(p=0.034)$ ], we found neither LOCS III nor LOD to be correlated with BMI [Spearman's Rho CC $=-0.008$ ( $p=0.943)$ and $-0.062(p=0.560)$, respectively] (Fig. 1b). Nevertheless, age was found to be associated with LOCS III [Spearman rho CC $=0.326(p=0.002)$ ] among our population cohort.

\section{Discussion}

The present findings confirm that obesity as defined by BMI is not associated with objective readings of crystalline LOD or subjective measures of LOCS III grading. Obesity is generally believed to accelerate ARC [1-3]. Although few evidence support the protective role of obesity against the cataract formation process $[25,26]$, several prospective observational studies have confirmed a positive longitudinal association between obesity and different subtypes of cataract [3].

In particular, the nonassociation of LOCS III and crystalline LOD readings with BMI from our own experience contrasts with the positive correlation of crystalline lens densitometry and obesity that was recently reported by Acer et al. [27]. Such a discrepancy is quite interesting considering that the adult population of our study with older age and higher levels of exposure to numerous cataractogenic environmental factors should have, at least in theory, reinforced the already positive correlation of crystalline lens density and BMI that was observed among children [27]. Aside from the difference in age of the population and the smaller sample size, failure to adjust for or measure the time spent outdoors (or the other equivalents of sun exposure) in the study by Acer et al. may also explain their discrepant findings. These authors demonstrated significantly higher Pentacam HR crystalline lens densitometry in obese children aged between 7 and 14 years compared to their age-matched lean controls. However, consistent with our findings, obese children aged between 15 and 18 years surprisingly displayed a comparable crystalline lens density to their control nonobese counterparts [27].

Kaya [28] and Watson et al. [29] suggested that the rise in growth hormone levels during puberty to induce hyperglycemia is counterbalanced by the antidiabetic actions of hyperinsulinemia in obese adolescents. As nonobese adolescents are more likely to be normoglycemic, both obese and nonobese adolescents may experience similar plasma glucose levels during puberty [28]. We speculate that a conditionally related mechanism may decelerate the characteristic progression of crystalline lens aging in older individuals to counteract with the adverse effects imposed by obesity and preserve the neutral metabolic status of crystalline lens. In addition, crystalline lens is an isolated island with no surrounding vessels and is suspended in a tightly regulated media. The crystalline lens could therefore act as an autonomous region by taking minimal effect from diverse metabolic regulations in obese and 
nonobese individuals and resulting in nonassociation with various degrees of obesity in adult individuals. By contrast, obese children before puberty may have a persistently elevated blood glucose which potentially results in nonenzymatic glycation of the lens proteins and a significantly higher crystalline lens density compared to nonobese nonpubescent children [28].

Despite these suggestions and from a molecular and submolecular standpoint, obesityinduced inflammation contributes to telomere length shortening in major systemic conditions, and a considerable number of age-related ocular pathologies have underlying systemic associations $[3,30]$. Telomere length shortening has been observed in lens epithelial cells and is closely associated with ARC [31]. These observations suggest a positive association of obesity and ARC (and thus, crystalline lens densitometry). Furthermore, subjects with higher BMI likely engage in a more sedentary lifestyle, with a less intensive physical activity that could result in a lower amount of sun exposure and a subsequently reduced risk of progression to cataract. Taken together, clinical endpoint studies are urgently required to confirm direction and magnitude of the correlation between lens density and obesity.

Along with the strengths, such as concurrent assessment of LOCS III and LOD by Pentacam HR in a homogenous group of healthy individuals, several limitations of our work should be considered including the relatively small population and the cross-sectional measurement of BMI. To explore the detrimental role of obesity on cataractogenesis, long-term investigation of BMI and metabolic status prior to the onset of crystalline lens changes is highly warranted.

Currently, little data supports whether the potential correlation of obesity and crystalline LOD is confined to children, adolescents, and younger individuals or is not affected by the chronological age. Determining the true nature of this correlation is particularly of interest largely due to the controversial role of obesity in ARC; so far that obesity has been proposed as a protective prognostic factor against the formation of ARC in several studies [25, 26]. Ideally, the conglomerate of risk factors potentially confounding the association between crystalline lens density and obesity, namely diabetes, nutrition, smoking, and sun exposure [26], should be carefully scrutinized in future related research.

\section{Acknowledgment}

The study is partly supported by Tehran University of Medical Sciences (Grant no. 11925).

\section{Disclosure Statement}

The authors have no conflicts of interest to disclose. The authors have no financial or proprietary interest in a product, method, or material described herein.

\section{References}

1 Cheung N, Wong TY: Obesity and eye diseases. Survey of ophthalmology. 2007;52:180-195.

2 Lee D-S, Han K, Kim H-A, et al: The gender-dependent association between obesity and age-related cataracts in middle-aged Korean adults. PloS One 2015;10:e0124262.

3 Pan C-W, Lin Y: Overweight, obesity, and age-related cataract: a meta-analysis. Optom Vis Sci 2014;91:478483.

4 Chovatiya R, Medzhitov R: Stress, inflammation, and defense of homeostasis. Mol Cell 2014;54:281-288.

5 Reddy PY, Giridharan NV, Balakrishna N, Validandi V, Pullakhandam R, Reddy GB: Increased risk of cataract development in WNIN-obese rats due to accumulation of intralenticular sorbitol. IUBMB Life 2013;65:472478. 
6 Radzevičienė L, Ostrauskas R: Body mass index, waist circumference, waist-hip ratio, waist-height ratio and risk for type 2 diabetes in women: a case-control study. Public H ealth 2013;127:241-246.

7 Muoio DM, Newgard CB: Obesity-related derangements in metabolic regulation. Annu Rev Biochem 2006;75: 367-401.

8 Spiegelman D, Israel RG, Bouchard C, Willett WC: Absolute fat mass, percent body fat, and body-fat distribution: which is the real determinant of blood pressure and serum glucose? Am J Clin Nutr 1992;55:10331044.

9 Leske MC, Wu S-Y, Hennis A, et al: Diabetes, hypertension, and central obesity as cataract risk factors in a black population: the Barbados Eye Study. Ophthalmology 1999;106:35-41.

10 Younan C, Mitchell P, Cumming R, Rochtchina E, Panchapakesan J, Tumuluri K: Cardiovascular disease, vascular risk factors and the incidence of cataract and cataract surgery: the Blue Mountains Eye Study. Ophthalmic Epidemiol 2003;10:227-240.

11 Sabanayagam C, Wang JJ, Mitchell P, et al: Metabolic syndrome components and age-related cataract: the Singapore Malay eye study. Invest Ophthalmol Vis Sci 2011;52:2397-2404.

12 Paunksnis A, Bojarskiene F, Cimbalas A, Cerniauskiene L, Luksiene D, Tamosiunas A: Relation between cataract and metabolic syndrome and its components. Eur J Ophthalmol 2007;17:605-614.

13 Galeone C, Petracci E, Pelucchi C, Zucchetto A, La Vecchia C, Tavani A: Metabolic syndrome, its components and risk of age-related cataract extraction: a case-control study in Italy. Ann Epidemiol 2010;20:380-384.

14 Klein BE, Klein R, Lee KE, Jensen SC: Measures of obesity and age-related eye diseases. Ophthalmic Epidemiol 2001;8:251-262.

15 Chylack LT, Wolfe JK, Singer DM, et al: The lens opacities classification system III. Arch Ophthalmol 1993;111: 831-836.

16 Kirkwood BJ, Hendicott PL, Read SA, Pesudovs K: Repeatability and validity of lens densitometry measured with Scheimpflug imaging. J Cataract Refract Surg 2009;35:1210-1215.

17 Zare MA, Mehrjardi HZ, Afarideh M, Bahrmandy H, Mohammadi S-F: Visual, keratometric and corneal biomechanical changes after Intacs SK implantation for moderate to severe keratoconus. J Ophthalmic Vis Res 2016; 11:17.

18 Pei X, Bao Y, Chen Y, Li X: Correlation of lens density measured using the Pentacam Scheimpflug system with the Lens Opacities Classification System III grading score and visual acuity in age-related nuclear cataract. Br J Ophthalmol 2008;92:1471-1475.

19 Klein BE, Hubbard L, Ferrier NJ, et al: Detecting progression of nuclear sclerosis by using human grading versus semiautomated computer grading. Invest Ophthalmol Vis Sci 2005;46:1155-1162.

20 Grewal DS, Brar GS, Grewal SPS: Correlation of nuclear cataract lens density using Scheimpflug images with Lens Opacities Classification System III and visual function. Ophthalmology 2009;116:1436-1443.

21 Davies NP, Morland AB: Color matching in diabetes: optical density of the crystalline lens and macular pigments. Invest Ophthalmol Vis Sci 2002;43:281-289.

22 Berendschot TT, Broekmans WM, Klöpping-Ketelaars IA, Kardinaal AF, van Poppel G, van Norren D: Lens aging in relation to nutritional determinants and possible risk factors for age-related cataract. Arch Ophthalmol 2002;120:1732-1737.

23 Robman L, Taylor H: External factors in the development of cataract. Eye 2005;19:1074-1082.

24 Dragomirescu V, Hockwin 0: Rotating slit image camera TOPCON SL 45. New developments for simultaneous image acquisition by photographic and CCD-assisted on-line documentation. Ophthalmic Res 1996;28(suppl 2):102-108.

25 Athanasiov PA, Casson R, Sullivan T, et al: Cataract in rural Myanmar: prevalence and risk factors from the Meiktila Eye Study. Br J Ophthalmol 2008;92:1169-1174.

26 Park S, Kim T, Cho S-I, Lee E-H: Association between cataract and the degree of obesity. Optom Vis Sci 2013; 90:1019-1027.

27 Acer S, Ağladıoğlu SY, Pekel G, et al: Density of the crystalline lens in obese and nonobese children. J AAPOS 2016;20:54-57.

28 Kaya A: Comment on: Density of the crystalline lens in obese and nonobese children. J AAPOS 2016;20:379.

29 Watson G, Baker LD, Cholerton BA, et al: Effects of insulin and octreotide on memory and growth hormone in Alzheimer's disease. J Alzheimers Dis 2009;18:595-602.

30 Tzanetakou IP, Katsilambros NL, Benetos A, Mikhailidis DP, Perrea DN: “Is obesity linked to aging?": adipose tissue and the role of telomeres. Ageing Res Rev 2012;11:220-229.

31 Babizhayev MA, Vishnyakova KS, Yegorov YE: Telomere-dependent senescent phenotype of lens epithelial cells as a biological marker of aging and cataractogenesis: the role of oxidative stress intensity and specific mechanism of phospholipid hydroperoxide toxicity in lens and aqueous. Fundam Clin Pharmacol 2011;25: 139-162. 


\section{Erratum}

In the research article by Mohammadi et al., "Obesity and Density of the Crystalline Lens: Revisiting a Growing Dilemma", Biomed Hub 2017;2:454979 (DOI: 10.1159/000454979), the following authors also contributed to the article: Mercede Majdi, Sahar Tabatabavakili, and Amir Houshang Beheshtnejad. The complete author list should read as follows:

Seyed-Farzad Mohammadia Sara Mirhadia Hadi Z. Mehrjardia, b Mohsen Afarideha Sahar Tabatabavakilia Mercede Majdia Amir Houshang Beheshtnejad ${ }^{a}$

aEye Research Center, Farabi Eye Hospital, Tehran University of Medical Sciences, and bStudents' Scientific Research Center, Tehran University of Medical Sciences, Tehran, Iran 